\title{
Lesões Desportivas na Natação
}

\section{Sports Injuries in Swimming}

Patrícia Raquel Carvalho de Aguiar² Fábio do Nascimento Bastos ${ }^{2}$ Jayme Netto Júnior? Luiz Carlos Marques Vanderlei 1,2 Carlos Marcelo Pastre ${ }^{1,2}$

1. Departamento de Fisioterapia. Faculdade de Ciências e Tecnologia/ Unesp. Presidente Prudente, SP Brasil.

2. Programa de Mestrado em Fisioterapia. Faculdade de Ciências e Tecnologia/Unesp. Presidente Prudente, SP - Brasil.

\section{Endereço para correspondência:} Universidade Estadual Paulista Unesp

Rua Roberto Simonsen, 305, Centro Educacional

19060-900 - Presidente Prudente, SP - Brasil

E-mail: praquel3@yahoo.com.br

\section{RESUMO}

O estudo objetivou analisar a ocorrência de lesões em nadadores, associando-as a fatores de risco específicos da modalidade e do atleta. Fizeram parte desse estudo 215 atletas, de ambos os sexos, participantes dos principais campeonatos promovidos pela Federação Aquática Paulista. Os dados foram obtidos por meio de Inquérito de Morbidade Referida, constituído por perguntas relacionadas ao atleta, modalidade e referentes ao tipo, mecanismo e local da lesão. A análise entre variáveis antropométricas e presença de lesão foi realizada pelo teste $t$ de Student ou pelo teste não paramétrico de Mann Whitney. Para relação entre as especificidades e o tipo, mecanismo e local da lesão, utilizou-se o teste de Goodman. Resultados significantes foram obtidos entre os atletas lesionados com mais idade e anos de treinamento. Segundo o mecanismo da lesão, o volume dos treinos é a principal causa de ocorrências de lesões e as tendinopatias são as lesões mais comuns. O ombro é o local mais acometido pelas diferentes especialidades, com exceção dos nadadores de peito que referiram a virilha. Conclui-se a partir dos achados que a exposição dos nadadores a prática esportiva associada ao volume de treinamento estão relacionadas com as frequentes lesões nestes atletas.

Palavras-chave: natação, lesões em atletas, fatores de risco.

\begin{abstract}
The study aimed to analyze the occurrence of injuries in swimmers, linking them to specific risk factors of the sport and the athlete. The present study evaluated 215 athletes of both sexes participating in the major championships, sponsored by the Aquatic Federation of São Paulo. Data were collected through a Morbidity Survey, consisting of questions related to the athlete, modality, as well as injury type, mechanism and site. Analysis between anthropometric variables and injury presence was performed by Student's t test or nonparametric Mann Whitney test. Goodman test was used for determination of specific relationship between injury type and site. Significant results were obtained between the older injured athletes and years of training. According to the injury mechanism, the training volume is the main cause of injury and tendinopathy are the most common examples. The shoulder is the site most affected by the different specialties, except for the breaststroke swimmers who reported the groin. The findings here state that exposure of swimmers to sports practice associated with the training volume is related to the frequent injuries in these athletes.
\end{abstract}

Keywords: swimming; injuries in athletes; risk factors.

\section{INTRODUÇÃO}

A exposição constante a modalidades esportivas diversas em quaisquer níveis de performance, por si, constitui-se situação de risco para ocorrência de lesões ${ }^{(1)}$. Estudos abordam o tema Lesões Desportivas $(\mathrm{LD})^{(2-4)}$; contudo, nem sempre há consenso sobre métodos de registro de informações ou, ainda, sobre aspectos conceituais relacionados ao assunto e, assim, maior atenção sobre a questão deve ser considerada.

Dentre os esportes acometidos por LD está a natação. Tal modalidade é popular em diversos países, sendo indicada para manutenção da saúde, tanto do sistema cardiorespiratório quanto do musculoesquelético(5) Contudo, a exposição à sua prática tem mostrado alguns riscos, sobretudo para integridade do sistema musculoesquelético ${ }^{(6)}$.

Os fatores causais para lesões têm sido caracterizados no âmbito biomecânico; contudo, existem limitações importantes para obtenção de visão epidemiológica mais evidente sobre o tema em questão(7). Inicialmente, deve-se atentar para a dificuldade de acesso às informações sobre os atletas e suas lesões conforme descreve Pastre ${ }^{(8)}$. Conseguir informações desta natureza não constitui tarefa fácil, o que fica evidenciado pela escassez de estudos disponíveis na literatura científica.
Outro fator importante é a escassez de informações referentes à associação entre lesões e especificidade dentro da modalidade em um universo amplo. O que se observa é a busca pela relação entre um gesto e uma lesão específica ${ }^{(6,9,10)}$. Dados sobre a gravidade de lesão também são expostos ${ }^{(11)}$, mas sem consenso, principalmente metodológico que se refira ao conceito. Ainda em relação ao método, identifica-se ampla variedade entre formas de análise de dados que varia desde análise descritiva até abordagens quantitativas mais complexas ${ }^{(6,12-14)}$

Levando em conta o exposto, este estudo justifica-se pela possibilidade de reunir informações de atletas de natação, praticantes dos variados estilos (borboleta, costa, peito, crawl) e provas (50, 100, 200, 400, 800 e 1.500 metros), e também provenientes de clubes e treinos diferentes. Destaca-se que o conhecimento da causa, mecanismo de lesão, fatores de risco, entre outros aspectos, pode auxiliar os profissionais da saúde no processo de prevenção, diagnóstico e tratamento desses agravos, o que contribui de forma importante para as ciências da saúde e do esporte.

Assim, constitui-se como objetivo deste estudo analisar a ocorrência de lesões em nadadores, associando-a a fatores de risco específicos da modalidade e do atleta, a partir de Inquérito de Morbidade Referida. 


\section{MÉTODOS}

\section{Delineamento observacional e população do estudo}

O estudo caracteriza-se como observacional e analítico. Foram analisados dados de 215 nadadores, sendo 80 do sexo feminino e 135 do masculino, selecionados aleatoriamente, os quais eram participantes dos principais campeonatos promovidos pela Federação Aquática Paulista (FAP). Destes atletas, 50 eram velocistas e 38 fundistas do estilo de crawl, 44 do estilo de borboleta, 40 do estilo de costas e 43 peitistas.

Como critério de exclusão, os atletas não poderiam ter menos de 18 anos, não assinar o Termo de Consentimento Livre e Esclarecido, terem menos de dois anos de prática do esporte em questão, e serem lesionados em outros momentos que não relacionados aos treinamentos e/ou competições de natação.

Após o consentimento dos dirigentes da FAP para realização da pesquisa, foram abordados os técnicos dos respectivos atletas com o intuito de aprovação. Todos os atletas foram esclarecidos sobre a pesquisa e autorizaram sua participação no estudo, mediante leitura e compreensão do Termo de Consentimento Livre e Esclarecido. O projeto referente ao estudo foi aprovado pelo Comitê de Ética em Pesquisa da FCT/Unesp-Presidente Prudente.

A coleta foi realizada durante as competições e, por se tratarem de eventos importantes para os participantes, a abordagem aos mesmos foi realizada em momentos distantes do período de preparação, como aquecimentos ou depois da sua prova, sem interferência no processo de recuperação ou concentração para realização de seu evento. Tal condição é sugerida por Pastre et al.(4).

\section{Técnicas e procedimentos}

Os dados foram obtidos por meio de Inquérito de Morbidade Referida, utilizando como ferramenta para coleta um questionário construído a partir do modelo de Pastre et al.(4) e adaptado para atender condições especificas do esporte ${ }^{(8)}$.

Para garantir a fidedignidade dos dados coletados foi realizado um piloto com 20 atletas. As principais mudanças ocorreram nos fatores relacionados ao tipo (muscular, osteoarticular e tendinopatias), e mecanismo (volume, intensidade, impacto direto ou impulsivo e atividades complementares) das lesões, adaptados à realidade do ambiente atlético dos nadadores. Foram avaliados o tipo, local anatômico e mecanismos da(s) lesão(ões) e optou-se por solicitar informações retroativas sobre a última temporada dos treinos e competições ${ }^{(4)}$.

\section{Descrição do instrumento de coleta}

O questionário utilizado foi elaborado por meio de modelo fechado e contendo dados relativos aos atletas (sexo, idade, peso, estatura, IMC, tempo de prática esportiva em anos, horas e quilometragem de treinamento por semana, dominância lateral e prova em que é especialista) e dados referentes às lesões (tipo de lesão, local anatômico e mecanismo causador).

Para efeito de estudo, considerou-se LD qualquer dor ou afecção musculoesquelética resultante de treinamentos e competições e que foi suficiente para causar alterações no treinamento normal, seja na forma, duração, intensidade ou frequência, conforme já utilizado em outras pesquisas ${ }^{(4,8)}$.

No sentido de facilitar a análise e apresentação dos resultados, as variáveis foram subdivididas em categorias a partir de agrupamentos para representar blocos mais expressivos de resultados sem, no entanto, modificar a essência de sua origem ou as conclusões do estudo.

Em relação à variável especialidade foram considerados atletas velocistas os especialistas nas provas que compreendem 50, 100 e 200 metros, e os fundistas, especialistas em provas de 400, 800 e 1.500 metros.
A variável tipo de lesão foi organizada em três grupos: lesão muscular, tendinopatias e lesões osteorticulares. Foi considerada lesão muscular qualquer agravo nesta estrutura, como distensão, contratura, mialgia ou miosite. As tendinopatias continham agravos como tendinite, tendinose, lesão tendínea ou outros danos específicos deste tecido. Para as lesões osteoarticulares foram agrupadas as lesões que acometiam estruturas ósseas e articulares como entorse, luxação, fraturas, sinovite e lesões condrais ${ }^{(15,16)}$.

Para determinação do local anatômico da lesão foi disponibilizada aos atletas uma figura ilustrativa do corpo humano no sentido de facilitar a identificação. Foram consideradas as regiões cervicais, torácicas e lombares, ombro, braço, cotovelo, punho e mão, pelve, coxa, virilha, joelho, perna, tornozelo e pé.

No inquérito, o mecanismo de lesão é caracterizado pelo tipo de atividade atlética em que os sinais e sintomas apareceram ou se acentuaram. Dessa forma, o mecanismo da lesão foi dividido nas principais condições causadoras entre os nadadores, sendo "elevado volume" (caracteriza-se por treinos para ganho de resistência e gesto esportivo excessivamente repetitivo), "elevada intensidade" (atividade de elevada velocidade e gesto esportivo excessivamente rápido e explosivo), "impacto direto ou impulsivo" (gestos específicos de saída, virada e chegada), "atividades complementares" (atividades não aquáticas, mas que estão presentes no plano de treino e ajudam no desempenho dos nadadores, como a musculação, alongamento, treinos de coordenação, de saltos e corda elástica).

\section{Análise estatística}

Para análise dos dados, inicialmente foi verificado se existia relação entre dominância lateral e sexo quanto à presença e ausência de lesão. Para esta análise foi utilizado o odds ratio e intervalo de confiança de $95 \%$. Como os resultados não apresentaram diferenças significantes, as análises foram feitas sem distinção de dominância lateral e sexo.

Para comparação das variáveis entre os atletas com presença ou ausência de lesão, inicialmente foi determinada a normalidade dos dados por meio do teste de Kolmorov e Smirnov. Quando a distribuição normal foi aceita, o teste $t$ de Student para amostras independentes foi aplicado (peso e altura) e nas situações em que a distribuição normal não foi aceita, foi aplicado o teste de Mann Whitney.

O estudo da associação entre tipo de lesão, mecanismo e local anatômico em cada especialidade, utilizou-se o teste de Goodman para contrastes entre e dentro de populações multinomiais.

Foram utilizadas letras minúsculas e maiúsculas para indicar as diferenças significativas entre os níveis do(s) fator(es) em comparação. A sistemática de leitura deve ser a seguinte: i) letras minúsculas (comparação entre letras dentro de uma coluna): usadas para comparação dos níveis do fator colocado nas linhas. ii) letras maiúsculas (comparação entre letras dentro de uma linha): usadas para comparação dos níveis do fator colocado nas colunas.

A interpretação das letras (maiúsculas ou minúsculas) deve ser feita considerando que letras iguais mostram resultados que não são estatisticamente diferentes. Todas os testes foram feitos com nível de significância de 5\%.

\section{RESULTADOS}

Dos atletas entrevistados, 177 referiram-se como destros, 100 com presença de lesão e 77 ausência, e, dos 38 canhotos, 21 referiram lesão e 17 não (odds ratio: 0,94, IC: 0,46-1,92). Em relação ao sexo, dos 80 atletas do sexo feminino, 51 referiram lesão e 29 não, e, dos 135 do sexo masculino, 70 referiram lesão e 65 não (odds ratio: 1,63, IC: 0,93-2,88).

As variáveis antropométricas e de treinamento segundo a presença de lesão, independentem da especialidade do participante. 
Na tabela 1 observam-se valores maiores para todas as variáveis dos lesionados em relação aos não lesionados. As diferenças estatísticas são notadas para idade e anos de prática esportiva.

Tabela 1. Distribuição das variáveis pessoais e de treinamento dos participantes segundo a presença ou ausência de lesão.

\begin{tabular}{|c|c|c|}
\hline \multirow{2}{*}{ Variável } & \multicolumn{2}{|c|}{ Lesão } \\
\hline & Ausente & Presente \\
\hline Idade & $\begin{array}{c}19,76 \pm 2,79 \\
(19)\end{array}$ & $\begin{array}{c}21,19 \pm 3,63 \\
(20) *\end{array}$ \\
\hline Peso & $\begin{array}{c}70,02 \pm 10,36 \\
(70)\end{array}$ & $\begin{array}{c}71,34 \pm 11,83 \\
(70,5)\end{array}$ \\
\hline Estatura & $\begin{array}{c}1,76 \pm 0,08 \\
(1,76)\end{array}$ & $\begin{array}{c}1,77 \pm 0,10 \\
(1,77)\end{array}$ \\
\hline IMC & $\begin{array}{c}22,54 \pm 2,02 \\
(22,39)\end{array}$ & $\begin{array}{c}22,64 \pm 1,95 \\
(22,82)\end{array}$ \\
\hline Anos de prática & $\begin{array}{c}11,83 \pm 4,30 \\
\quad(12)\end{array}$ & $\begin{array}{c}13,77 \pm 4,88 \text { \# } \\
(14)\end{array}$ \\
\hline Frequência semanal & $\begin{array}{c}7,40 \pm 1,90 \\
\text { (8) }\end{array}$ & $\begin{array}{c}7,84 \pm 1,66 \\
\text { (8) }\end{array}$ \\
\hline Quilometragem semanal & $\begin{array}{c}43,23 \pm 12,31 \\
(45)\end{array}$ & $\begin{array}{c}43,07 \pm 10,40 \\
(42)\end{array}$ \\
\hline
\end{tabular}

*Diferença significante em relaçăo à mediana da idade dos participantes que não referiram lesão $(p<0,01)$. \# Diferença significante em relação à média dos anos de prática dos participantes que não referiram lesão $(p<0,01)$.

De acordo com a tabela 2, a mediana de idade de 21 anos para os fundistas lesionados foi significantemente maior em comparação aos atletas não lesionados. Nas outras modalidades observou-se distribuição casual.

Tabela 2. Distribuição segundo idade na ausência e presença de lesão nas diferentes modalidades atléticas.

\begin{tabular}{|c|c|c|}
\hline \multirow{2}{*}{ Especialidade } & \multicolumn{2}{|c|}{ Lesão } \\
\hline & Ausente & Presente \\
\hline Velocistas & $\begin{array}{c}20,05 \pm 2,68 \\
19\end{array}$ & $\begin{array}{c}21,00 \pm 3,90 \\
19\end{array}$ \\
\hline Fundistas & $\begin{array}{c}18,71 \pm 2,37 \\
18\end{array}$ & $\begin{array}{c}21,88 \pm 4,33 \\
21^{*}\end{array}$ \\
\hline Borboleta & $\begin{array}{c}20,62 \pm 3,10 \\
20\end{array}$ & $\begin{array}{c}20,35 \pm 2,79 \\
20\end{array}$ \\
\hline Costas & $\begin{array}{c}18,87 \pm 2,28 \\
18\end{array}$ & $\begin{array}{c}20,25 \pm 2,71 \\
19,50\end{array}$ \\
\hline Peito & $\begin{array}{c}20,50 \pm 3,01 \\
20\end{array}$ & $\begin{array}{c}22,31 \pm 3,27 \\
20\end{array}$ \\
\hline
\end{tabular}

Para todas as modalidades, com exceção para o nado de peito, os valores mostram-se maiores para os que sofreram algum tipo de agravo. Houve diferença estatisticamente significante para os fundistas (mediana) e para os especialistas do estilo costas (média) em relação aos anos de prática esportiva (tabela 3).

Para os velocistas do estilo crawl e peito houve maiores taxas de lesões musculares e tendíneas em relação às osteoarticulares. Para as outras especialidades não foi notada diferença. A comparação entre as especialidades em cada tipo de lesão, também mostrou distribuição casual (tabela 4).
Tabela 3. Distribuição das medidas descritivas dos anos de prática segundo a ausência e presença de lesão nas diferentes modalidades atléticas.

\begin{tabular}{c|c|c}
\hline \multirow{2}{*}{ Especialidade } & \multicolumn{2}{|c}{ Lesão } \\
\cline { 2 - 3 } & Ausente & Presente \\
\hline \multirow{2}{*}{ Velocistas } & $12,70 \pm 4,70$ & $14,40 \pm 4,34$ \\
14,50 & 14 \\
\hline Fundistas & $9,47 \pm 2,58$ & $16,29 \pm 5,58$ \\
& 10 & $14 *$ \\
\hline Borboleta & $12,43 \pm 4,57$ & $12,24 \pm 4,57$ \\
\hline Costas & 12 & $13,58 \pm 3,74 \#$ \\
& $10,37 \pm 3,76$ & 13 \\
\hline Peito & 10 & $13,33 \pm 5,47$ \\
& $14,12 \pm 4,57$ & 13 \\
\hline
\end{tabular}

* Diferença significante em relação à mediana de prática dos participantes que năo referiram lesão $(p<0,05)$. \# Diferença significante em relação à média de prática dos participantes que năo referiram lesão $(p<0,05)$.

Tabela 4. Distribuição de frequências absoluta e relativa (\%) do tipo de lesão segundo modalidade atlética.

\begin{tabular}{|c|c|c|c|}
\hline \multirow{2}{*}{ Especialidade } & \multicolumn{3}{|c|}{ Tipo de lesão } \\
\hline & Lesão muscular & Osteoarticular & Lesão tendínea \\
\hline Velocistas & $\begin{array}{c}28(37,29) \mathrm{a} \\
\mathrm{B}\end{array}$ & $\begin{array}{c}6(10,17) \text { a } \\
A\end{array}$ & $\begin{array}{c}31(52,54) \mathrm{a} \\
\mathrm{B}\end{array}$ \\
\hline Fundistas & $\begin{array}{c}8(24,24) a \\
A\end{array}$ & $\begin{array}{c}6(18,18) \text { a } \\
A\end{array}$ & $\begin{array}{c}19(57,58) \mathrm{a} \\
\mathrm{A}\end{array}$ \\
\hline Borboleta & $\begin{array}{c}13(39,39) \text { a } \\
\text { A }\end{array}$ & $\begin{array}{c}5(15,15) \mathrm{a} \\
\mathrm{A}\end{array}$ & $\begin{array}{c}15(45,45) \mathrm{a} \\
\mathrm{A}\end{array}$ \\
\hline Costas & $\begin{array}{c}7(20,00) \mathrm{a} \\
\mathrm{A}\end{array}$ & $\begin{array}{c}8(22,86) \mathrm{a} \\
\mathrm{A}\end{array}$ & $\begin{array}{c}20(57,14) \mathrm{a} \\
\mathrm{A}\end{array}$ \\
\hline Peito & $\begin{array}{c}23(42,59) a \\
B\end{array}$ & $\begin{array}{c}7(12,96) \mathrm{a} \\
\mathrm{A}\end{array}$ & $\begin{array}{c}24(44,44) a \\
B\end{array}$ \\
\hline
\end{tabular}

Letras maiúsculas comparam os tipos (colunas) dentro de cada especialidade (linhas) (A < B e letras minúsculas comparam especialidades dentro de cada tipo $(\mathrm{a}<\mathrm{b})$.

Os resultados do tipo do mecanismo de lesão em cada modalidade atlética estão apresentados na tabela 5. Para os velocistas de crawl, o volume de treino $(59,32 \%)$ e as atividades complementares $(32,20 \%)$ foram significantemente mais frequentes que a intensidade de esforço (0\%). Além disso, o volume de trabalho, também apresentou diferença significante para o impacto direto ou impulsivo (8,47\%). Para os nadadores fundistas e os velocistas especialistas nos estilos borboleta e peito, o volume de trabalho foi o mecanismo mais referido e apresentou diferença estatisticamente significante em comparação aos outros mecanismos. Para os nadadores de costas a maioria das lesões ocorreram pelo volume de treino $(57,14 \%)$ que apresenta diferença significante em relação à intensidade (1,85\%) e impacto (1,85\%).

Quando comparados os mecanismos de lesão em relação às especialidades, identificou-se que para o volume de trabalho houve distribuição relativa significantemente maior no estilo peito $(90,74 \%)$ em relação aos nadadores de costas $(57,14 \%)$ e velocistas de crawl (59,32\%) e os fundistas de crawl (87,88\%) em relação aos velocistas (59,32\%). Para o mecanismo atividades complementares houve diferença significante entre os velocistas $(32,20 \%)$ em relação aos nadadores de peito $(5,56 \%)$ e casual entre os outros estilos.

A tabela 6 descreve a distribuição dos locais anatômicos referidos em ocorrências de lesões, segundo a especialidade. Destaca-se o ombro como o local com maior ocorrência de lesão, com exceção ao estilo peito, que apresentou a virilha como local mais acometido. 
Tabela 5. Distribuição de frequências absoluta e relativa (\%) do mecanismo de lesão segundo modalidade atlética.

\begin{tabular}{|c|c|c|c|c|}
\hline \multirow{2}{*}{ Especialidade } & \multicolumn{4}{|c|}{ Mecanismo de Lesão } \\
\hline & Volume & Intensidade & Impacto & Ativ. comp. \\
\hline Velocista & $\begin{array}{c}35(59,32) a \\
C\end{array}$ & $\begin{array}{c}0(0,00) \mathrm{a} \\
\mathrm{A}\end{array}$ & $\begin{array}{c}5(8,47) a \\
A B\end{array}$ & $\begin{array}{c}19(32,20) b \\
B C\end{array}$ \\
\hline Fundista & $\begin{array}{c}29(87,88) b c \\
\text { B }\end{array}$ & $\begin{array}{c}0(0,00) a \\
A\end{array}$ & $\begin{array}{c}1(3,03) \mathrm{a} \\
\mathrm{A}\end{array}$ & $\begin{array}{c}3(9,09) a b \\
\text { A }\end{array}$ \\
\hline Borboleta & $\begin{array}{c}24(72,73) a b c \\
\text { B }\end{array}$ & $\begin{array}{c}2(6,06) \mathrm{a} \\
\mathrm{A}\end{array}$ & $\begin{array}{c}0(0,00) a \\
A\end{array}$ & $\begin{array}{c}7(21,21) a b \\
A\end{array}$ \\
\hline Costas & $\begin{array}{c}20(57,14) a b \\
B\end{array}$ & $\begin{array}{c}2(5,71) a \\
A\end{array}$ & $\begin{array}{c}3(8,57) \mathrm{a} \\
\mathrm{A}\end{array}$ & $\begin{array}{c}10(28,57) a b \\
A B\end{array}$ \\
\hline Peito & $\begin{array}{c}49(90,74) \mathrm{C} \\
\text { B }\end{array}$ & $\begin{array}{c}1(1,85) \mathrm{a} \\
\mathrm{A}\end{array}$ & $\begin{array}{c}1(1,85) \mathrm{a} \\
\mathrm{A}\end{array}$ & $\begin{array}{c}3(5,56) a \\
A\end{array}$ \\
\hline
\end{tabular}

Letras maiúsculas comparam os mecanismos (colunas) dentro de cada especialidade (linhas) $(A<B)$ e letras minúsculas, comparam especialidades dentro de cada mecanismo $(a<b)$

Tabela 6. Distribuição de frequências, absoluta e relativa (\%) da modalidade atlética segundo local anatômico acometido.

\begin{tabular}{|c|c|c|c|c|c|}
\hline \multirow{2}{*}{$\begin{array}{c}\text { Local } \\
\text { anatômico }\end{array}$} & \multicolumn{5}{|c|}{ Especialidades } \\
\hline & Velocista & Fundistas & Borboleta & Costas & Peito \\
\hline Cervical & $\begin{array}{c}2 \\
(3,39)\end{array}$ & $\begin{array}{c}0 \\
(0,00)\end{array}$ & $\begin{array}{c}0 \\
(0,00)\end{array}$ & $\begin{array}{c}0 \\
(0,00)\end{array}$ & $\begin{array}{c}0 \\
(0,00)\end{array}$ \\
\hline Torácica & $\begin{array}{c}3 \\
(5,08)\end{array}$ & $\begin{array}{c}1 \\
(3,03)\end{array}$ & $\begin{array}{c}1 \\
(3,03)\end{array}$ & $\begin{array}{c}2 \\
(5,71)\end{array}$ & $\begin{array}{c}4 \\
(7,41)\end{array}$ \\
\hline Lombar & $\begin{array}{c}9 \\
(15,25)\end{array}$ & $\begin{array}{c}4 \\
(12,12)\end{array}$ & $\begin{array}{c}6 \\
(18,18)\end{array}$ & $\begin{array}{c}5 \\
(14,29)\end{array}$ & $\begin{array}{c}7 \\
(12,96)\end{array}$ \\
\hline Braço & $\begin{array}{c}3 \\
(5,08)\end{array}$ & $\begin{array}{c}2 \\
(6,06)\end{array}$ & $\begin{array}{c}0 \\
(0,00)\end{array}$ & $\begin{array}{c}0 \\
(0,00)\end{array}$ & $\begin{array}{c}1 \\
(1,85)\end{array}$ \\
\hline Cotovelo & $\begin{array}{c}1 \\
(1,69)\end{array}$ & $\begin{array}{c}3 \\
(9,09)\end{array}$ & $\begin{array}{c}2 \\
(6,06)\end{array}$ & $\begin{array}{c}3 \\
(8,57)\end{array}$ & $\begin{array}{c}6 \\
(11,11)\end{array}$ \\
\hline Punho/mão & $\begin{array}{c}0 \\
(0,00)\end{array}$ & $\begin{array}{c}1 \\
(3,03)\end{array}$ & $\begin{array}{c}0 \\
(0,00)\end{array}$ & $\begin{array}{c}2 \\
(5,71)\end{array}$ & $\begin{array}{c}0 \\
(0,00)\end{array}$ \\
\hline Ombro & $\begin{array}{c}28 \\
(47,46)\end{array}$ & $\begin{array}{c}17 \\
(51,52)\end{array}$ & $\begin{array}{c}16 \\
(48,48)\end{array}$ & $\begin{array}{c}12 \\
(34,29)\end{array}$ & $\begin{array}{c}11 \\
(20,37)\end{array}$ \\
\hline Pelve & $\begin{array}{c}2 \\
(3,39)\end{array}$ & $\begin{array}{c}0 \\
(0,00)\end{array}$ & $\begin{array}{c}0 \\
(0,00)\end{array}$ & $\begin{array}{c}0 \\
(0,00)\end{array}$ & $\begin{array}{c}3 \\
(5,56)\end{array}$ \\
\hline Coxa & $\begin{array}{c}3 \\
(5,08)\end{array}$ & $\begin{array}{c}1 \\
(3,03)\end{array}$ & $\begin{array}{c}0 \\
(0,00)\end{array}$ & $\begin{array}{c}1 \\
(2,86)\end{array}$ & $\begin{array}{c}3 \\
(5,56)\end{array}$ \\
\hline Virilha & $\begin{array}{c}0 \\
(0,00)\end{array}$ & $\begin{array}{c}0 \\
(0,00)\end{array}$ & $\begin{array}{c}0 \\
(0,00)\end{array}$ & $\begin{array}{c}0 \\
(0,00)\end{array}$ & $\begin{array}{c}12 \\
(22,22)\end{array}$ \\
\hline Joelho & $\begin{array}{c}8 \\
(13,56)\end{array}$ & $\begin{array}{c}2 \\
(6,06)\end{array}$ & $\begin{array}{c}5 \\
(15,15)\end{array}$ & $\begin{array}{c}5 \\
(14,29)\end{array}$ & $\begin{array}{c}6 \\
(11,11)\end{array}$ \\
\hline Perna & $\begin{array}{c}0 \\
(0,00)\end{array}$ & $\begin{array}{c}0 \\
(0,00)\end{array}$ & $\begin{array}{c}0 \\
(0,00)\end{array}$ & $\begin{array}{c}1 \\
(2,86)\end{array}$ & $\begin{array}{c}0 \\
(0,00)\end{array}$ \\
\hline $\begin{array}{c}\text { Tornozelo/ } \\
\text { pé }\end{array}$ & $\begin{array}{c}0 \\
(0,00)\end{array}$ & $\begin{array}{c}2 \\
(6,06)\end{array}$ & $\begin{array}{c}3 \\
(9,09)\end{array}$ & $\begin{array}{c}4 \\
(11,43)\end{array}$ & $\begin{array}{c}1 \\
(1,85)\end{array}$ \\
\hline
\end{tabular}

\section{DISCUSSÃO}

Dos 215 atletas avaliados, 121 relataram ao menos uma lesão durante a temporada corrente.Tais achados concordam com estudos de Rodeo ${ }^{(9)}$ e Chen et al. ${ }^{(17)}$ que verificaram elevada frequência de lesões na natação. Contudo, a exposição constante associada ao elevado volume de trabalho, como demonstrado neste trabalho, parece ser fator determinante para tal ocorrência. Segundo Rodeo ${ }^{(9)} \mathrm{e}$ Pink e Tibone(18), atletas competitivos podem nadar cerca de 10 a
$14 \mathrm{~km}$ por dia, durante seis a sete vezes por semana, isso equivale aproximadamente a 2.500 braçadas em um único dia, o que contribui para alto risco de lesões.

Observa-se que a relação entre sexo e domínio lateral com ocorrência de lesão teve distribuição casual, sem diferença estatística significante, o que concorda com os achados no estudo Weldon e Richardson ${ }^{(19)}$, tanto para questões de gênero como da dominância lateral, e discorda de outros estudos de mesma natureza que constataram maior frequência de lesão entre as mulheres quando comparadas aos homens ${ }^{(20,21)}$.

As características antropométricas e de treinamento foram investigadas e nota-se relação entre os anos de prática esportiva e a idade dos participantes com as ocorrências de lesão. Para os especialistas do estilo costas e fundistas, houve relação entre maior exposição e ocorrência de lesões analisando a variável anos de prática. Por outro lado, a faixa etária dos participantes não permite hipóteses relacionadas ao envelhecimento, como as levantadas por Faulkner et al.(22) e Maharam et al.(23) que incluem perda de flexibilidade, força e potência muscular, devido à faixa etária da amostra desta pesquisa.

Em relação ao mecanismo de lesão, no presente estudo, o volume dos treinos é o principal causador de lesões referidas pelos atletas. Weldon e Richardson ${ }^{(19)}$ e Banks et al. ${ }^{(12)}$ relatam que o volume do treinamento é responsável pela maioria das lesões em nadadores, sendo a principal causa de ausências em competições e treinamentos. No estudo de González-Boto et al. ${ }^{(24)}$ observou-se que, quando os atletas atingiam o volume máximo nos treinos, ocorriam aumentos significativos nas escalas de estresse relacionados às lesões e exaustão emocional, com diminuição dos valores relacionados ao sucesso, recuperação física e autoeficiência na escala de recuperação.

Explorações relacionadas ao tipo de lesão também foram feitas. Nota-se, para os participantes desta pesquisa, que as tendinopatias são as mais citadas, independente da especialidade, o que corrobora os achados de alguns autores ${ }^{(19,25)}$. As razões abordadas para ocorrência de tal lesão são relacionadas comumente ao excesso de repetições associados ao desequilíbrio de força muscular ${ }^{(12,13,19,25)}$. As lesões musculares também foram referidas de maneira significante e, segundo alguns autores ${ }^{(13,25)}$, estão associadas com o mesmo fator causal das tendinopatias, reiterando repetição e desequilíbrio muscular.

Em relação aos locais anatômicos, observa-se que o ombro foi o mais referido entre todas as especialidades, exceto para o nado de peito. Tais achados também são relatos por Chalmers e Morrison ${ }^{(7)}$, que descrevem os nadadores de crawl, borboleta e costas como sendo os mais acometidos por lesões neste seguimento.

Biomecanicamente, Yanai et al. ${ }^{(26)}$ relataram que a estrutura articular do ombro dos nadadores sofrem com o uso repetitivo, e a sobrecarga a estas estruturas ocorre principalmente durante a entrada da mão na água, em que o ângulo de elevação do ombro atinge o seu máximo e também durante a fase de recuperação da braçada, na qual há o excesso de rotação interna do mesmo.

Ainda concordando com os achados deste estudo, Capaci et al.(27) verificaram que, dos nadadores competitivos do sexo masculino que relataram lesão musculoesquelética, a maioria referiu dor no ombro seguida da coluna lombar e a articulação do joelho, respectivamente. Os autores(27) ainda descrevem que a dor no ombro ocorreu com frequência nos nadadores de crawl e a dor do joelho foi observada somente nos peitistas.

Em relação aos resultados do estilo peito, nota-se que, comparado a outras especialidades, as lesões na virilha são mais frequentes, o que corrobora com o estudo de Grote et al.(28), que justificam tal fato, de forma genérica, devido aos movimentos repetitivos e a dinâmica de pernada deste estilo. 
Segundo Rodeo ${ }^{(9)}$, na especialidade de peito os esforços repetitivos nos músculos adutores da coxa durante o nado fazem com que ocorra estresse durante a finalização do trabalho de pernas, quando a adução forçada da coxa ocorre com os joelhos em valgo e os tornozelos e pés rodados externamente.

Os resultados permitem inserir elementos à literatura que são escassos, sobretudo em relação à análise de associação. Apesar de boa parte das conclusões ser observada na prática de campo, alguns dos fatores são discretos e passam despercebidos aos envolvidos diretos dentro da modalidade. Sugere-se que, a partir da vivência de campo nas coletas deste estudo, cada equipe possua dinâmica de registros sendo mais específica à realidade do treinamento, ou seja, descrevendo com riqueza de detalhes as lesões e seu processo de instalação, para que, desta forma, possam ser estabelecidos momentos de alerta relacionados ao treinamento e inseridos programas de prevenção específicos à cada situação.

\section{CONCLUSÃO}

A partir dos achados deste estudo, conclui-se elevada frequência de lesões em nadadores, sem distinção significante para dominância lateral e sexo. O maior valor médio para anos de prática esportiva é o fator de risco para instalação de lesões em fundistas e especialistas no estilo costas; e, em relação à idade, os maiores valores destacam-se entre os fundistas. $O$ elevado volume de trabalho é o mecanismo mais referido por todas as especialidades. $O$ tipo de lesão mais frequente é a tendinopatia, e o local anatômico mais referido pelos participantes é o ombro, exceto para os peitistas que referiram a virilha como o local anatômico mais acometido.

Todos os autores declararam não haver qualquer potencial conflito de interesses referente a este artigo.

\section{REFERÊNCIAS}

1. Auvinen JP, Tammelin TH, Taimela SP, Zitting PJ, Mutanen PO, Karppinen Jl. Musculoskeletal pains in relation to different sport and exercise activities in youth. Med Sci Sports Exerc 2008;40:1890-900

2. Bahr R, Holme I. Risk factors for sports injuries - a methodological approach. Br J Sports Med 2003;37:384-92.

3. Phillips LH. Sports injury incidence. Br J Sports Med 2000;34:133-6.

4. Pastre CM, Carvalho Filho G, Monteiro HL, Netto Júnior J, Padovani CR. Lesões desportivas no atletismo: comparação entre informações obtidas em prontuários e inquéritos de morbidade referida. Rev Bras Med Esporte 2004;10:1-8.

5. Tanaka H. Swimming exercise: impact of aquatic exercise on cardiovascular health. Spots Med 2009;39:377-87.

6. Richardson AB. Injuries in competitive swimming. Clin Sport Med 1999;18:287-91.

7. Chalmers DJ, Morrison L. Epidemiology of non-submersion injuries in aquatic sporting and recreational activities. Sports Med 2003;33:745-70

8. Pastre CM. Lesões Desportivas no atletismo: Comparação entre informações obtidas em prontuários e inquéritos de morbidade referida. [Dissertação]. São José do Rio Preto. Famerp; Faculdade de Medicina; 2003.

9. Rodeo SA. Knee pain in competitive swimming. Clin Sports Med 1999;18:379-87.

10. Seifert L, Chollet D. Modelling spacial-temporal and coordinative parameters in swimming. J Sci Med Sport 2009;12:495-9.

11. Parkkari J, Kannus P, Natri A, Lapinleimu I, Palvanen M, Heiskanen $M$, et al. Active living and injury risk. J Sci Med Sport 2004;25:209-16.

12. Banks KP, Ly JQ, Beall DP, Grayson DE, Bancroft LW, Tall MA. Overuse injuries of the upper extremity in the competitive athlete: magnetic resonance imaging findings associated with repetitive trauma. Curr Probl Diagn Radiol 2005;34:127-42.

13. Santos MJ, Belangero WD, Almeida GL. The effect of joint instability on latency and recruitment order of the shoulder muscles. J Electromyogr Kinesiol 2007;17:167-75.

14. Hangai M, Kaneoka K, Hinotsu S, Shimizu K, Okubo Y, Miyakawa S, et al. Lumbar intervertebral disk degeneration in athletes. Am J Sports Med 2009;37:149-55.
15. Peterson L, Renström P. Lesões do esporte: prevenção e tratamento. 3a ed. São Paulo: Manole; 2002. 16. Whiting WC, Zernicke RF. Biomecânica da lesão musculoesquelética. Rio de Janeiro: Guanabara Koogan; 2001.

17. Chen SK, Cheng YM, Lin YC, Hong YJ, Huang PJ, Chou PH. Investigation of management models in elite athletes injuries. Kaohsiung J Med Sci 2005;21:220-7.

18. Pink MM, Tibone JE. The painful shoulder in the swimming athlete. Orthop Clin Noth Am 2000;31:247-61.

19. Weldon EJ, Richardson AB. Upper extremity overuse injuries in swimming. A discussion of swimmer's shoulder. Clin Sports Med 2001;20:423-38.

20. Constantini NW, Warren M.P. Special problems of the female athlete. Baillières Clin Rheumatol 1994;8:119-219.

21. Hiemstra LA, Kirkley A. Shoulder instability in female athletes. Sports Med Arthroscopy Review 2002;10:50-7.

22. Faulkner JA, Davis CS, Mendias CL, Brooks SV. The aging of elite male athletes: age-related changes in performance and skeletal muscle structure and function. Clin J Sport Med 2008;18:501-7.

23. Maharam LG, Bauman PA, Kalman D, Skolnik H, Perle SM. Masters Athletes: factors affectting performance. Sports Med 1999;28:273-85.

24. González-Boto R, Salguero A, Tuero C, González-Gallego J, Márquez S. Monitoring the effects of training load changes on stress and recovery in swimmers. J Physiol Biochem 2008;64:19-26.

25. Rupp S, Berninger K, Hopf T. Shoulder problems in high level swimmers - impigement, anterior instability, muscular imbalance? Int J Sports Med 1995;16:557-62

26. Yanai T, Hay JG. Shoulder impingement in front-craw swimming: II analysis of stroke technique. Med Sci Sport Exerc 1998:32:30-40

27. Capaci K, Ozcaldiran B, Durmaz B. Musculoskeletal pain in elite competitive male swimmers. Pain Clin 2002;14:229-34

28. Grote K, Lincoln TL, Gamble JG. Hip adductor injury in competitive swimmers. Am J Sports Med 2004;32:104-8 